\title{
Improved algorithm based on StOMP for compressed sensing reconstruction
}

\author{
Fengjun Zhao \\ 1. Engineering Research Center of Digitized Textile \& \\ Apparel Technology, Ministry of Education \\ 2. College of Information Science and Technology \\ Donghua University, Shanghai 201620, P. R. China \\ Shanghai, China \\ zhaofengjun118@yeah.net \\ Kuangrong Hao* \\ 1. Engineering Research Center of Digitized Textile \& \\ Apparel Technology, Ministry of Education \\ 2. College of Information Science and Technology \\ Donghua University, Shanghai 201620, P. R. China \\ Shanghai, China \\ *krhao@dhu.edu.cn
}

\author{
Yongsheng Ding \\ 1. Engineering Research Center of Digitized Textile \& \\ Apparel Technology, Ministry of Education \\ 2. College of Information Science and Technology \\ Donghua University, Shanghai 201620, P. R. China \\ Shanghai, China \\ ysding@dhu.edu.cn
}

\begin{abstract}
1 Abstract-In order to solve the problem of low reconstruction reconstruction accuracy in stagewise orthogonal matching pursuit(StOMP), stagewise subspace orthogonal matching pursuit(StSOMP) was proposed and realized. In this work, backtracking was introduced to StOMP. First, according to StOMP algorithm to select atoms that meet the threshold condition, these atoms constitute the candidate set, then compute the least squares to eliminate the wrong atoms and find out new atoms to replace, gradually close to the real support set. The experimental results show that the proposed algorithm retains the quickness of StOMP algorithm, make up for the shortcomings of the low reconstruction accuracy. The algorithm performances better under the condition of the low sampling rate, and can meet the fast and accurate reconstruction of large size image.
\end{abstract}

Keywords-compressed sensing; reconstruction algorithm; stagewise orthogonal matching pursuit algorithm

\section{INTRODUCTION}

Nyquist sampling theorem states that, in order to perfect reconstruction the analog signal, sampling frequency is at least twice the original signal. To eliminate the redundant information in the sampled data, it can achieve the purpose of

\footnotetext{
${ }^{1}$ This work was supported in part by the Key Project of the National Nature Science Foundation of China (No.61134009), the National Nature Science Foundation of China (Nos. 61473077, 61473078), Program for Changjiang Scholars from the Ministry of Education, Specialized Research Fund for Shanghai Leading Talents, Project of the Shanghai Committee of Science and Technology (No.13JC1407500),Innovation Program of Shanghai Municipal Education Commission (No. 14ZZ067), and the Fundamental Research Funds for the Central Universities (15D110423).

*Correspondence should be addressed to Kuang-rong HAO , E-mail: krhao@dhu.edu.cn
}

compression. However, for digital images and video, this sampling method will produce a large amount of sampling data, and storage and transmission costs will be greatly increased, it will result in a large waste of resources. Emmanuel and Candes proposed the compressed sensing ${ }^{[1][2] \text {, }}$ it points out that under the condition of far less than the Nyquist sampling rate, the discrete samples of the signal obtained by random sampling can be perfect reconstructed using the nonlinear reconstruction algorithm. Compressed sensing broke through the frequency limit of Nyquist sampling theorem, it can reconstruct the original image with few observations, which can greatly reduce the transmission bandwidth, image storage. Because of these advantages, compressed sensing is widely used in signal processing ${ }^{[3 \sim 5]}$.

Compressed sensing includes the sparsity, measurement and reconstruction. At present, the reconstruction algorithm mainly consists of convex optimization algorithm, greedy pursuit algorithm and combination algorithm ${ }^{[6]}$.The convex optimization algorithm needs less observations and its reconstruction accuracy is higher, but the time complexity is large. The combination algorithm is strict to the sample structure, and is subject to the constraints of hardware conditions. The greedy algorithm has simple structure, which greatly improving the speed of operation, but it sacrifices for a certain reconstruction precision. Reconstruction algorithm with low complexity and high reconstruction accuracy and small number of observations is the focus of the current research $^{[7]}$.

This paper mainly studies greedy pursuit algorithm. Orthogonal matching pursuit (OMP) algorithm has the advantages of simple structure and easy to implement ${ }^{[8]}$; Stagewise orthogonal matching pursuit (StOMP) algorithm 
performs faster than OMP, but the reconstruction accuracy is lower ${ }^{[9 \sim 10]}$; Regularized orthogonal matching pursuit (ROMP) algorithm which has good performance in large scale problems needs to know the signal sparsity ${ }^{[11212]}$;Subspace pursuit (SP) algorithm with low complexity needs to know the signal sparsity ${ }^{[13]}$; The performance of Sparsity adaptive matching pursuit (SAMP) algorithm is similar to that of the SP algorithm when sparsity is unknown, but its reconstruction speed is slow ${ }^{[13]}$.

In order to solve the contradiction between time complexity and accuracy, backtracking was introduced to stagewise orthogonal matching pursuit algorithm. This paper presents stagewise subspace orthogonal matching pursuit algorithm (StSOMP).

At first, this paper gives a theoretical model of compressed sensing and reconstruction algorithm, and then discusses the implementation process of StSOMP algorithm. Finally, the effectiveness of the algorithm is verified by simulation experiments.

\section{COMPRESSED SENSING THEOREM}

\section{A. Compressed sensing}

Assuming that we have a vector $x \in \mathfrak{R}^{n}$ which we expand in an orthogonal basis $\Psi$ as follow:

$$
x=\Psi \alpha
$$

where $\Psi$ is orthogonal basis and the signal $\alpha$ is $k$-sparse

According to the compressed sensing theorem, a matrix $\Phi \in \mathfrak{R}^{m \times n}(m<n)$ can be designed to reduce the dimension of the original signal:

$$
y=\Phi x=\Phi \Psi \alpha
$$

Where $y \in \mathfrak{R}^{m}$ is measurement vector, $\Psi$ is sensing matrix, $\Phi$ is irrelevant to $\Psi$. Since $m<n$, the equation has no definite solution. but due to the signal $\alpha$ is $k$ - sparse and $\Phi$ satisfies RIP, it is possible to solve the problem through $l_{0}$ norm. The estimated value of $\alpha$ is obtained by solving the minimum $l_{0}$ norm as follows:

$$
\alpha=\arg \min \|\alpha\|_{0} \quad \text { s.t. } \quad y=\Phi \Psi \alpha
$$

The common solution (3) method is a greedy pursuit algorithm, and each time one or more atoms is chosen to meet the satisfy conditions, and then update the support set, gradually approaching the true value. This paper studies the StOMP algorithm and SP algorithm.

\section{B. Stagewise Orthogonal Matching Pursuit Algorithm}

Stagewise Orthogonal Matching Pursuit Algorithm (StOMP) uses atom selection criteria of the OMP algorithm, compared with other matching pursuit algorithms, StOMP algorithm has the advantage of adaptive, that means in the case of sparsity unknown, the original signal can be approximated by step size.

The algorithm is as follows ${ }^{[13]}$ :

(1) Calculate the correlation coefficient of the residuals and the atom of the observation matrix, and find the atomic coordinates that satisfy the threshold conditions:

$$
B_{t}=\left\{j:\left|<r_{t-1}, \theta_{j}>\right|>\tau\left\|r_{t}\right\|_{2} / \sqrt{m}\right\}
$$

Where $r_{*}$ is the residual, $\theta_{j}$ is $j-t h$ atom, $\tau \in(2.5,3)$ is constant, $m$ is the length of measurement vector.

(2) Update support set $\Lambda_{t}=\Lambda_{t-1} \cup B_{t}$

(3) Judge whether it reaches the iteration: if reached, then stop the iteration; if not, according to the formula update residuals $r_{t}=y-\Theta_{\Lambda_{t}} \Theta_{\Lambda_{t}}^{-1} * y$, then repeat step(1)(2).

\section{Subspace Pursuit Algorithm(SP)}

The SP algorithm introduces the idea of backward tracing, and then replaces the atoms in the selected atom with the newly identified atom, and gradually approaches the real support set.

The algorithm is as follows:

(1) Calculate the correlation coefficient of the residuals and the atom of the observation matrix, and find the coordinates of $k$ most matched atoms:

$B_{t}=\max _{j}\left\{j:\left|<r_{t-1}, \theta_{j}>\right|, k\right\}$

(2) Build a candidate set: $C_{t}=\Lambda_{t-1} \cup B_{t}$

(3) Update support set: $\Lambda_{t}=\max \left\{\Theta_{C_{t}}^{-1} * y, k\right\}$

(4) Updated residuals: $r_{t}=y-\Theta_{\Lambda_{t}} \Theta_{\Lambda_{t}}^{-1} * y$

(5) Judge whether it reaches the iteration: if reached, then stop the iteration; if not, then repeat the iteration.

\section{Stagewise Subspace Orthogonal Matching Pursuit ALGORITHM}

StSOMP algorithm is an improved algorithm for StOMP which introduced the backtracking of SP into StSOMP. The algorithm utilizes the StOMP adaptive selective atomic, and the complexity of the algorithm is low. It combined with subspace tracking of thought, and improves the accuracy of the reconstruction, solves the problem of high precision and fast reconstruction based on sparse unknown degree.

The StSOMP algorithm proposed in this paper mainly includes three stages, that is the atomic primaries, the construction of candidate sets, and the update support set.

Initialization: the initial error $r_{0}=y$, the candidate set and the set of support are empty.

\section{A. Atomic primaries}

According to the idea of StOMP algorithm, the correlation coefficient of the residuals and each atom of the observed matrix are calculated, and the atomic coordinates that meet the threshold conditions are got. The set of primary atomic is $B_{t}=\left\{j:\left|<r_{t-1}, \theta_{j}>\right|>\tau\left\|r_{t}\right\|_{2} / \sqrt{m}\right\}$, and the number of atoms in the primaries is num . $_{\text {. }}$

\section{B. Build a candidate set}

Because the threshold is more suitable for the Gauss signal, it is not suitable for all signals. In the process of the algorithm, if there is no primary atom, it will lead to the support set can not be updated, the algorithm enters the death cycle. In order 
to guarantee the validity and reliability, this paper introduces decision the condition. If the primary atomic set is empty, indicating that threshold setting is not reasonable, and then choose the largest correlation coefficient of the atom as the primary atomic, and reduce the threshold; if primary atomic set is not empty, the primary atomic set are added to the candidate set.

\section{Update support set}

From $2 *$ num $_{t}$ atoms, num ${ }_{t}$ atoms are selected to make $\Theta_{C_{t}}^{-1} * y$ maximum, and then update the support set $\Lambda_{t}=\max \left\{\Theta_{C_{t}}^{-1} * y\right.$, num $\left._{t}\right\}$. The main purpose of this step is to remove the wrong atoms in the candidate set and replace them with the new ones. The aim is to make the estimation of the support set recursively, and ensure that the distance between the subspace and the measurement matrix is strictly decreasing.

TABLEI is the pseudo code of the algorithm. It shows that compared with greedy matching algorithm, the advantage of the algorithm is the reconstruction process using a threshold selection atomic, need not be known a priory sparsity, but the threshold setting is fit for Gaussian signals. In order to ensure the reliability, the control condition is joined, and backtracking is introduced to this paper. In order to ensure the rapidity at the same time and improve the reconstruction accuracy, half of the atoms are selected to add to the support set from the set of candidates each time.

TABLEI StSOMP Pseudo Code

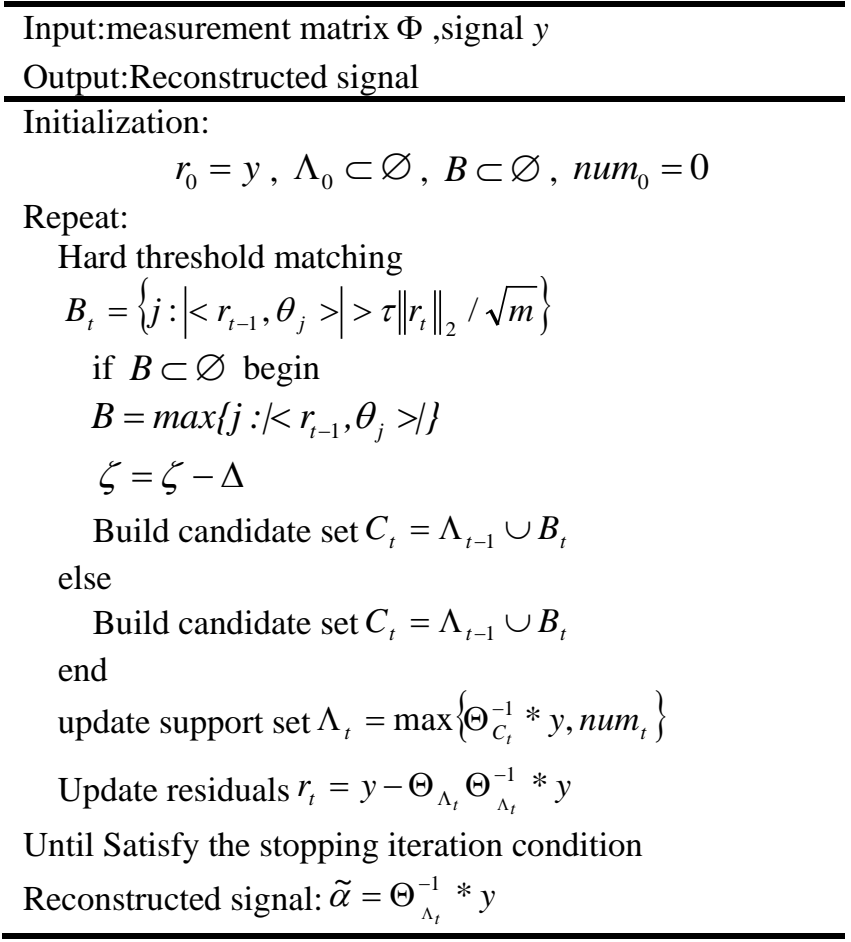

\section{EXPERIMENTAL ANALYSIS}

In order to verify the effectiveness of the algorithm, the performance of the StSOMP algorithm and the traditional greedy tracking algorithm BP, OMP, StOMP are compared in the reconstruction error, PSNR, running time using MATLAB programming. Experiment 1 mainly aimed at one dimensional signal with the known sparsity degree; In Experiment 2 mainly aimed at two dimensional images under different sample rate.

\section{A. Experiment 1}

Experimental signal is Gauss signal with length 200 and sparse degree 20, the observation matrix is Gauss random matrix and the sampling value $M=100$.Fig. 1 shows the error of all algorithms. It can be seen that the reconstruction error of OMP, BP algorithm is almost 0 , and the reconstruction error of StSOMP algorithm is near 0 , and the error of StOMP algorithm is large. Formula for reconstruction error is $\left\|x_{\text {rec }}-x\right\|_{2}^{2} /\|x\|_{2}^{2}$, where $x_{\text {rec }}$ is reconstructed signal, and $x$ is the original signal.On the other hand, Fig.2 shows the reconstruction time of all algorithms. It can be seen from 50 times experiment that BP is about ten times OMP, OMP is about 5 times StOMP, StSOMP is between OMP and StOMP.

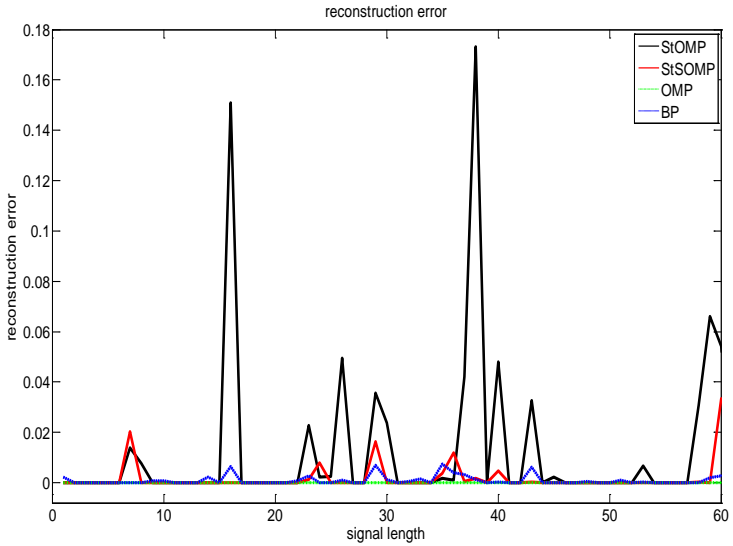

Fig. 1:Error curve of each algorithm

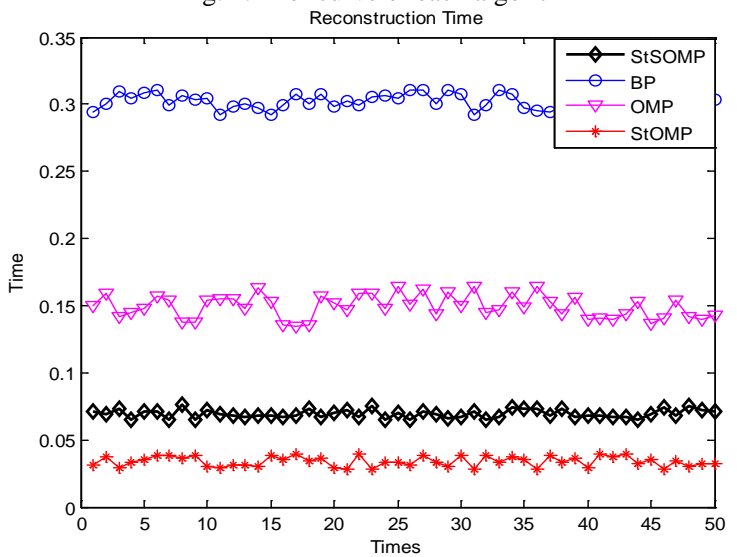

Fig.2: Reconstruction Time of Gauss Signal

\section{B. Experiment 2}

In this experiment, we studied the reconstruction of two dimensional images under different sampling rates. In order to ensure the sparsity of the signal, the standard experimental images using Lena 256*256 images, using Gauss random matrix as the measurement matrix. Since the original image is not sparse in time domain, the image is sparse by wavelet transform. TABLEII compares the performance of different algorithms in PSNR, the reconstruction error and the reconstruction time under different sampling rates. It can be 
seen that StSOMP has been improved in the reconstruction error and PSNR, compared with the BP algorithm has a smaller gap, but the reconstruction time is significantly less than the BP algorithm. Fig.3 shows the reconstruction effect of different methods at the sampling rate of 0.5 . It can be seen that the reconstruction effect of proposed algorithm and BP algorithm is good, the effect of StOMP reconstruction is poor, the effect of OMP reconstruction is between the two. Fig. 3(f) shows the details. From TABLE 3, it can be seen that this algorithm can meet the requirements of large images when the sampling rate is low and the time complexity is reduced significantly when the accuracy is satisfied.

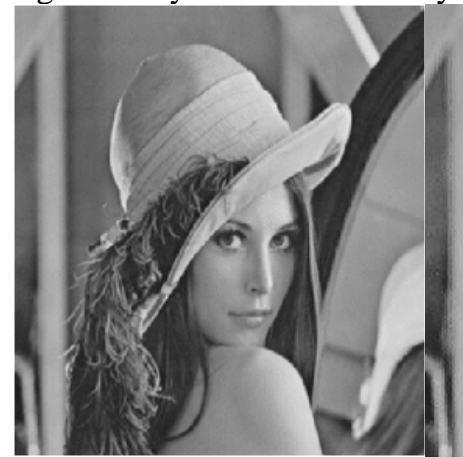

(a)original

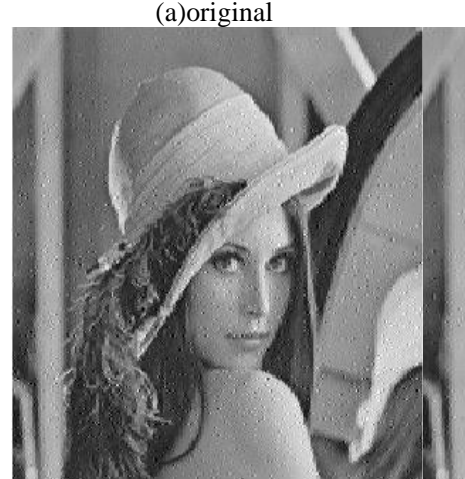

(c)OMP

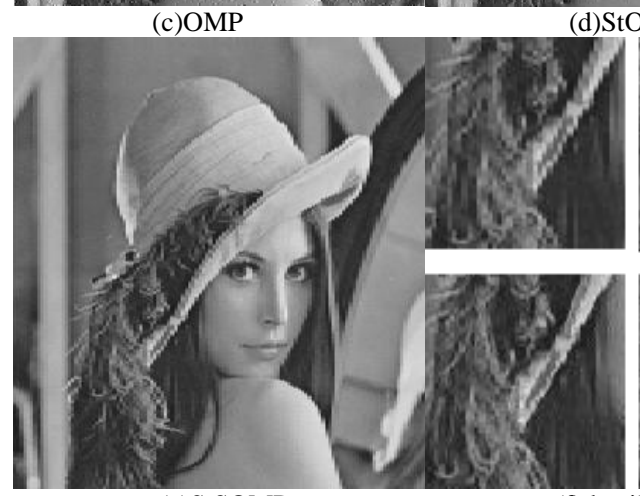

(e)StSOMP

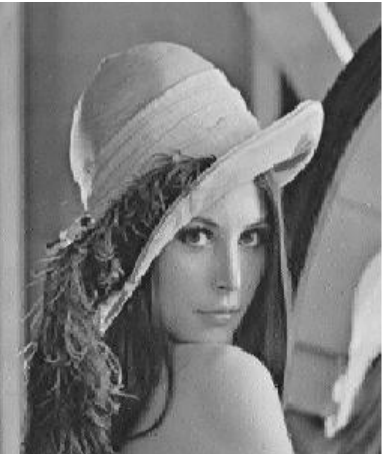

(b)BP

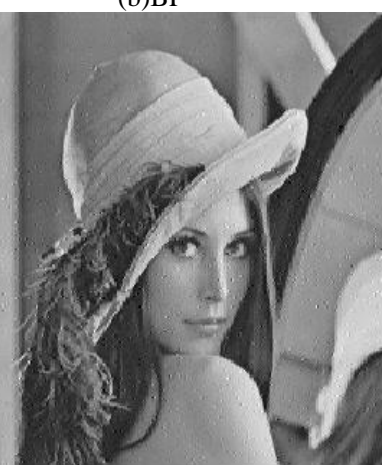

(d)StOMP

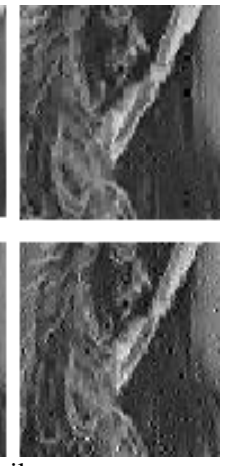

(f)detail

\section{CONCLUSIONS}

This paper analyzes the advantages and disadvantages of common greedy tracking algorithm, presents a StSOMP algorithm with fast and accurate reconstruction under the condition of sparsity unknown. The algorithm uses the hard threshold method of StOMP to solve the problem of sparse degree, and then backtracking is introduced, and according to the method of least square method to remove the wrong atoms in the candidate set and replace them with the new ones.The reconstruction precision is improved, and the contradiction between the traditional greedy algorithm in the reconstruction precision, sampling frequency and running time is solved. Although the greedy algorithm has great advantages in the reconstruction of the time, but the reconstruction precision is still to be improved, the next step is to study the reconstruction algorithm with fast and high accuracy.

\section{REFERENCES}

[1] CANDES E J, TAO T,Decoding by linear programming, IEEE Transactions on Information Theory, 2005, 51(12):4203-4215.

[2] DONOHO D L,Compressed sensing,IEEE Transactions on Information Theory, 2006, 52(4): 1289-1306.

[3] SUK,HAN G L,SUN H J,Anti-viewpoint changing image matching algorithm based onSURF,Chinese Journal of Liquid and Crystals and Display,2013,28(4):626-632.

[4] ZHAO R ZH,LIU $X$ Y,SUN MG,Wavelet denoising via sparse representation,Science Signal Information,2009,52(8):1371- 1377.

[5] ZHANG Y, WANG Y Y, Li W,Reconstruction photoacoustic image based on total variation method,optical precision engineering, 2012,01:204-212.

[6] Wei Di,Milenkovic O,Subspace Pursuit for Compressive Sensing Signal Reconstruction, IEEE Transactions on Information Theory, 2009, 55(5): 2230-2249.

[7] ZHAO Y J, ZHU Y W, SUN B,An improved sparse adaptive matching pursuit algorithm,Signal processing, 2012,01: 80-86.

[8] Karahanoglu N B,Erdogan H,A*orthogonal matching pursuit:Best-first search for compresses sensing signal recovery,Digital Signal Processing:A Review Journal,2012,22(4):555-568.

[9] ZHANG W H,An improvement on StOMP for sparse solution of linear underdetermined problems,Control Conference,2013:1951- 1956.

[10] Marsousi M,Abhari K,Babynp P,MULTI-STAGE OMP sparse coding using local matching pursuit atoms selection,Acoustics, Speech and Signal Processing,2013:(5):1783-1787.

[11] Liu Jing,Han ChongZhao,Han Feng,A novel compressed sensing based track before detect algorithm for tracking multiple targets,Information Fusion,2013(8):1514-1519.

[12] Liu Yaxin, Zhao Ruizhen, Hu Shaohai, Jiang Chunhui,A regularized adaptive matching pursuit tracking algorithm for compressed sensing signal reconstruction,electronic and information journal, 2010,11:27132717.

[13] Wu Di, Wang Kuimin, Zhao Yuxin,Piecewise regularized orthogonal matching pursuit algorithm,optics and precision engineering, 2014 (5):1395-1402.

TABLEII Comparison of the reconstruction performance

\begin{tabular}{c|ccc|cccc|cr}
\hline Sample Rate(M/N) & \multicolumn{3}{c|}{0.5} & \multicolumn{3}{c}{0.3} & \multicolumn{2}{c}{0.1} \\
\hline Algorithm & PSNR & Time/s & Error & PSNR & Time/s & Error & PSNR & Time/s & Error \\
\hline BP & 39.8695 & 6.8974 & 0.0678 & 37.1508 & 5.8800 & 0.0388 & 35.1681 & 4.9354 & 0.0540 \\
OMP & 36.3020 & 2.1114 & 0.0811 & 34.3331 & 0.9699 & 0.0588 & 33.6081 & 0.3030 & 0.0746 \\
StOMP & 30.5578 & 0.2023 & 0.0881 & 30.8006 & 0.2247 & 0.1168 & 32.8898 & 0.1587 & 0.1121 \\
StSOMP & 36.7012 & 0.9889 & 0.0640 & 35.6676 & 0.2142 & 0.0696 & 34.6661 & 0.1137 & 0.0840 \\
\hline
\end{tabular}

